\title{
Offline Shop Recommendation based On Online Shopping History
}

\author{
Chongji Mo ${ }^{a}$, Congcong Chen ${ }^{\mathrm{b}}$ \\ School of Software, Shanghai Jiaotong University, Shanghai 200240, China

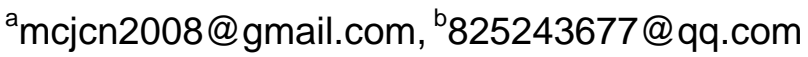

Keywords: Recommender system, O2O.

\begin{abstract}
Most existing POI recommender systems is facing the problem of sparsely of data. In this paper, an $\mathrm{O} 2 \mathrm{O}$ (Online to Offline) recommendation method is proposed, which can take advantage of users' online shopping history data. User behavior is converted to user preference, modeled as user and interest-term matrix. Based on the model, the result list of offline shop is recommended by O2O recommendation algorithm. The algorithm is implemented, and experiments on the real dataset show the feasibility of the method.
\end{abstract}

\section{Introduction}

In recent years, with the rapid development of online e-commerce shopping website such as TAOBAO, TMALL and 360BUY, traditional offline retail market is facing unprecedented challenges [1]. One important advantage of online shopping is its recommender systems, which can advise consumers with goods which they may be interested in. If the technology of recommender system can be applied to traditional offline retail market, both shopkeepers and users will benefit a lot. For example, if offline shop recommender system is applied, users will find their desirable shops more easily; meanwhile, shops will also usher in more potential customers.

However, it is hard to recommend offline shop [2], due to following reasons:

First, offline POI (Point of Interest) recommender systems usually use spatial location data, which is sparse and irregular [3].

Secondly, input of offline recommender system is implicit feedback, which indirectly reflect opinion through observing user behavior [4].

Thirdly, according to Ebbinghaus forgetting curve, users' interest changes over time [5]. Therefore it is difficult to model the user preference.

\section{Related work}

Recommender system. Most existing recommender systems can be classified into following categories [6]: collaborative filtering (CF, including User-based $\mathrm{CF}$ and Item-based CF [7]), contended-based filtering, hybrid algorithm [8], Matrix Factorization (MF) and rank based algorithm [9]. Different recommendation algorithms are chosen due to the property of the input data. Amazon has put Item-based CF into practice [7], so do many websites in mainland, China, such as TAOBAO, YOUKU, etc. MF-based recommendation algorithms are also widely used, which are thought perform efficiently on many famous datasets, such as Movie Lens [10].

Implicit feedback. A common task of recommender system is to improve customer experience through personalized recommendations based on prior implicit feedback [4]. As explicit feedback (e.g. user's rating to a movie) is not always available, recommenders should infer user preference from the more abundant implicit feedback, which indirectly reflect opinion through observing user behavior [11]. Types of implicit feedback include purchase history, browsing history, search patterns, or even mouse movements. Many research work in the recommendation field is focused on processing explicit feedback, while in most situations, recommender systems face implicit feedback. 


\section{Offline Shop Recommendation - an 020 approach}

It is hard to recommend offline shop. Meanwhile, recommender systems are widely used in online shopping websites. One Important reason is, in the network environment, rich user behaviors are collected. The comparison of online user behavior (online data) and spatial location data (offline data) is listed as follow:

Table 1 Online/Offline data comparing

\begin{tabular}{c|cc}
\hline Feature & Online data & Offline data \\
\hline Clarity & Yes & No \\
Noise & No & Yes \\
Semantic information & Immediate & Require datasets \\
\hline
\end{tabular}

Clarity means that user behavior is easy to explain. When users are viewing the shopping website, their behavior can be classified to browse, purchase and comment. In contrast, spatial location data can only tell the position (longitude and latitude) and the duration time. Efforts remain to be made in order to analyze the user behavior. Noise means that since spatial location is collected by different devices like cell phones and IPads, the result may not be so accurate, thus contains noise, which may cause deviation while analyzing the user preference. Semantic information means while the data contains semantic information of the shop or the goods, which helps to find user interest. Semantic information can be immediately collected through shopping websites. For example, online product titles contain information of brand, type, material of the goods. To achieve the same aim, spatial location data must be used together with other datasets like local POI data.

Therefore, online user behavior has certain advantages over spatial location data when analyzing user preference. In this study, O2O recommender system is proposed, in order to take advantage of online shopping data. The basic step is shown in the following figure:
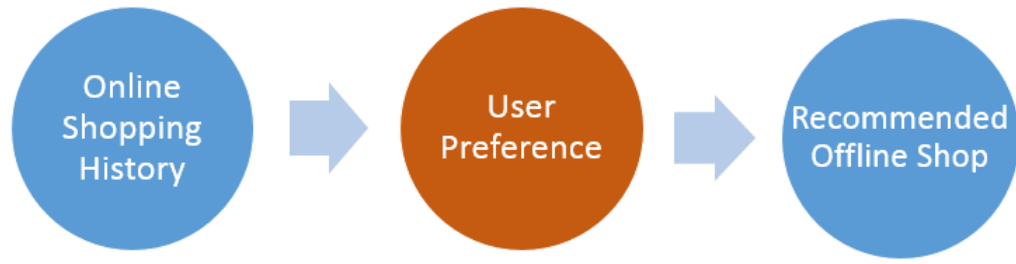

Fig. 1 O2O recommendation step

The input is users' online shopping history, which contains users' behavior (browse, collect or purchase) and semantic information. The input is analyzed and user preference is modeled. According to the user preference model, recommender systems is going to recommend offline shops. Here, user preference model works as a bridge between the input and the output. Following chapters introduce these steps in detail.

\section{User Preference Model}

Item to interest-term. Let $I$ be a collection of item. Let $T$ be a collection of interest-term. $T$ Contains all possible interest-terms taken from the titles or names of items, which contain the semantic information (descriptions). Then it adopts the bag of words model to represent items as vectors. Each item $i \in I$ is represented by a bag of interest-term.

EXAMPLE 1 . Let $i$ be a product which may be browsed or purchased by users. The title of $i$ is 'Korean-style white women dresses'. Let $T$ be a vocabulary contains: 'Korean-style, Chinese-style, white, red, yellow, black, dresses, jean, T-shirt'. Then $i$ can be represented by 3 interest-terms: Korean-style, white, dresses'.

Behavior convert to score. Let $U$ be a collection of user. Let $M$ be a collection of shopping behaviors of all users. Let $A$ be a collection of all actions user may process in one behavior. Each $m \in M$ is represented as $m\left(u_{m}, i_{m}, a_{m}, d_{m}\right)$, which means behavior $m$ takes between user $u_{m}$ and itemi $i_{m}$, and the action is $a_{m} . d_{m}$ Represents the number of days passed between the action time and current time. Different action (browse, purchase, etc.) shows different degrees of interest the user has 
towards the item. Therefore, each action $a \in A$ has an interest score $s_{a}$. The final score from user $u$ towards item $i$ is represented byr $r_{\text {ui }}$, and calculated by the following model ( $\propto$ is recession factor, which shows that the effect of such behavior becomes weak as time with time):

$$
r_{u i}=\sum_{m_{\mathrm{n}} \in M}\left(s_{a_{m_{0}}} \times \alpha^{d_{m_{0}}}\right)
$$

User interest-term model. Snice each item $i$ can be represented by several interest-terms, and the score between certain user $u$ and item $i$ can be calculated, now we can model the user preference by user interest-term model. The rating between certain user $u$ and interest-term $t \in T$ is correlated to the score between $u$ and such item $i$ that $t$ is one of its interest-term (represented as $i \rightarrow t$ ). The rating between user $u$ and interest-term $t, r_{u t}$ is calculated by:

$$
r_{u t}=\sum_{i \in I}^{i \rightarrow t} r_{u i}=\sum_{i \in I}^{i \rightarrow t} \sum_{m_{n} \in M}^{u_{m_{0}}=u, i m_{m_{0}}=i}\left(s_{a_{m_{0}}} \times \alpha^{d_{m_{0}}}\right)
$$

EXAMPLE 2. Let $U$ be $\{$ Alice, Peter, Ben... $\}, T$ be $\{$ Korean-style, white, dresses... $\}$, and given certain $I, M, A, \alpha$, then the user preference is modeled as user interest-term matrix. Each element $r_{u t}$ in the matrix can be calculated by (2). An instance of user interest-term matrix is shown in Table 2.

Table 2 user interest-term matrix instance

\begin{tabular}{c|cccc}
\hline & Korean-style & white & dresses & $\ldots$ \\
\hline Alice & 25.1 & 28.4 & 57.9 & $\ldots$ \\
Peter & 0 & 21.3 & 0 & $\ldots$ \\
Ben & 0 & 76.7 & 5.1 & \\
$\ldots$ & $\ldots$ & $\ldots$ & $\ldots$ & $\ldots$ \\
\hline
\end{tabular}

\section{O2O recommendation algorithm}

Based on the above user preference model, we now present the pseudo-code of $\mathrm{O} 2 \mathrm{O}$ recommendation algorithm in Algorithm 1.

\section{Algorithm 1: The O2O Recommendation Algorithm}

Input: A collection of user's behaviors $M$, a collection of item $I$ which contain title, the recession factor $\propto$, a collection of action score $S_{[A]}$, and a matrix of shop and interest-term $P_{[o]_{\ell} \mid T_{0} \|}$.

Output: each user's offline shop list.

$1 \quad U \leftarrow$ ExtractUsers $(M)$

$2 \quad T \leftarrow$ ExtractTerms $(I)$

3 Initial $R_{|U|,|T|}$, set elements be 0

4 for each $m \in M$

5 for each $t$, as $i_{m} \rightarrow t$

$6 \quad r_{u t}+=s_{a_{m}} \times \alpha^{d_{m_{0}}}$

$7 \quad P_{|o|,|T|} \leftarrow$ UnifyTerms $\left(P_{|O|_{\imath}\left|T_{0}\right|}\right)$

$8 Q_{|U|,|0|} \leftarrow R \times P^{T}$

9 for each row in $Q_{|U|, \mid} O \mid$

10 sort shops $o \in O$ by $q_{u o}$

Line 1 extracts the user collection. Line 2 extracts the set of interest-term $T$, which is the overall vocabulary of users, items and shops. Line 4-6 compose $R_{|U|_{\mid}|T|}$. Line 7 unifes the vocabulary of shops $T_{0}$ in order to match the vocabulary of users $T$. $O$ Is the collection of shops? The intermediate of above algorithm, $R_{|U|,|T|}$ is user interest-term matrix, as we mentioned in previous chapter. 


\section{Experiments}

The above algorithm is implemented and experimented. We set $\propto$ to 0.99 to simulate the real human interest decline. We use the Rec-Tmall [12] dataset, which is announced by Alibaba. 658 interest-terms is generated from titles of 562 products. 836 users and 658 interest-terms makes a matrix of 550088 elements. The user preference model is generated from 7557 user behaviors. The recommendation result make sense because it is easy to explain and understand since user interest-term matrix contains intuitive semantic message. The performance of the result matches with our expectations, and therefore the method is feasible.

As an interesting discovery, we list the top frequency interest-terms as follows: 2014, Korean-style, female, new, slimmer, and trendy. It seems that most users in the dataset are probably young ladies.

\section{Future prospects}

It seems that $\mathrm{O} 2 \mathrm{O}$ recommender system has broad business prospects, as $\mathrm{O} 2 \mathrm{O}$ is considered to be the gospel of traditional retail market. However, still a lot of efforts should be made to put it into industry practice. The realization of ideal future requires the support of owners of data.

\section{References}

[1] Z. Huang, D. Zeng and H. Chen, “A Comparison of Collaborative-Filtering Recommendation Algorithms for E-commerce”, IEEE Intelligent Systems 22 (2007), p. 68-78.

[2] Ricci F, Rokach L, Shapira B. Introduction to recommender systems handbook [M]. Springer US, 2011.

[3] Sarwat, Mohamed, et al. "LARS*: An Efficient and Scalable Location-Aware Recommender System." IEEE Transactions on Knowledge and Data Engineering (2013): p.1-1

[4] Hu Y, Koren Y, Volinsky C. Collaborative filtering for implicit feedback datasets[C]//Data Mining, 2008. ICDM'08. Eighth IEEE International Conference on. IEEE, 2008: p.263-272.

[5] Tobias Betz, Lawrence Cabac, Michael Duvigneau, Thomas Wagner and Matthias WesternEbbinghaus: Software Engineering with Petri Nets: A Web Service and Agent Perspective. T. Petri Nets and Other Models of Concurrency (TOPNOC) 9: p.41-61 (2014)

[6] G. Adomavicius, A. Tuzhilin. Toward the Next Generation of Recommender Systems: A Survey of the State-of-the-Art and Possible Extensions,IEEE Trans, on Knowl. And Data Eng., vol. 17, iss. 6, p. 734-749, 2005.

[7] Linden G, Smith B, York J. Amazon. Com recommendations: Item-to-item collaborative filtering [J]. Internet Computing, IEEE, 2003, 7(1): p.76-80.

[8] Melville P, Mooney R J, Nagarajan R. Content-boosted collaborative filtering for improved recommendations[C]//AAAI/IAAI. 2002: p.187-192.

[9] Jiankai Sun, Shuaiqiang Wang, Byron J. Gao and Jun Ma: Learning to rank for hybrid recommendation. CIKM 2012: p.2239-2242

[10] Information on http://grouplens.org/datasets/movielens/

[11] D.W. Oard and J. Kim, "Implicit Feedback for Recommender Systems", Proc. 5th DELOS Workshop on Filtering and Collaborative Filtering, p. 31-36, 1998.

[12] Information on http://tianchi.alibaba.com/. 